\title{
Extreme Preservers of Zero-term Rank Sum over Fuzzy Ma- trices
}

\author{
SEOK-Zun Song* And YeOn-Jung NA \\ Department of Mathematics, Jeju National University, Jeju 690-756, Republic of \\ Korea \\ e-mail: szsong@jejunu.ac.kr and s1zzang@jejunu.ac.kr
}

ABstract. In this paper, we consider two extreme sets of zero-term rank sum of fuzzy matrix pairs:

$$
\begin{gathered}
z_{1}(\mathcal{F})=\left\{(X, Y) \in \mathcal{M}_{m, n}(\mathcal{F})^{2} \mid z(X+Y)=\min \{z(X), z(Y)\}\right\} \\
z_{2}(\mathcal{F})=\left\{(X, Y) \in \mathcal{M}_{m, n}(\mathcal{F})^{2} \mid z(X+Y)=0\right\}
\end{gathered}
$$

We characterize the linear operators that preserve these two extreme sets of zero-term rank sum of fuzzy matrix pairs.

\section{Introduction and preliminaries}

During the last century, problems on the characterization of the linear operators that leave certain matrix subsets invariants were actively studied. For survey of these types of problems, we refer to the article of Song $([7])$ and the papers in $[6]$. The specified frame of problems is of interest both for matrices with entries from a field and for matrices with entries from an arbitrary semiring such as Boolean algebra, nonnegative integers, and fuzzy semiring. It is necessary to note that there are several rank functions over a semiring that are analogues of the classical function of the matrix rank over a field. Detailed research and self-contained information about rank functions over semirings can be found in [1] and [7].

There are some results on the inequalities for the rank function of matrices([1], [2], [3] and [4]). Beasley and Guterman ([1]) investigated the rank inequalities of matrices over semirings. And they characterized the equality cases for some rank inequalities in [2]. The investigation of linear preserver problems of extreme cases of the rank inequalities of matrices over fields was obtained in [4]. The structure of matrix varieties which arise as extremal cases in the inequalities is far from being understood over fields, as well as semirings. A usual way to generate elements of such a variety is to find a matrix pairs which belongs to it and to act on this set by various linear operators that preserve this variety. Song and his colleagues $([3])$

* Corresponding Author.

Received August 26, 2010; accepted October 6, 2010.

2000 Mathematics Subject Classification: 15A86, 15A04, 15A33.

Key words and phrases: Linear operator, zero-term rank, fuzzy semiring, fuzzy matrix. 
characterized the linear operators that preserve the extreme cases of column rank inequalities over semirings. There are some results on the linear operators that preserve zero-term $\operatorname{rank}([5])$.

In this paper, we characterize linear operators that preserve the sets of matrix pairs which satisfy extreme cases for the zero term rank inequalities for the sum of matrices over fuzzy semirings.

Definition 1.1. A semiring $\mathcal{S}$ consists of a set $\mathcal{S}$ and two binary operations, addition and multiplication, such that:

- $\mathcal{S}$ is an Abelian monoid under addition (identity denoted by 0 );

- $\mathcal{S}$ is a semigroup under multiplication (identity, if any, denoted by 1 );

- multiplication is distributive over addition on both sides;

- $s 0=0 s=0$ for all $s \in \mathcal{S}$.

Definition 1.2. A semiring is called antinegative if the zero element is the only element with an additive inverse.

Definition 1.3. A semiring is called chain if the set $\mathcal{S}$ is totally ordered with universal lower and upper bounds and the operations are defined by $a+b=\max \{a, b\}$ and $a \cdot b=\min \{a, b\}$.

It is straightforward to see that any chain semiring is commutative and antinegative.

Throughout we assume that $m \leq n$. The matrix $I_{n}$ is the $n \times n$ identity matrix, $J_{m, n}$ is the $m \times n$ matrix of all ones, $O_{m, n}$ is the $m \times n$ zero matrix. We omit the subscripts when the order is obvious from the context and we write $I, J$, and $O$, respectively. The matrix $E_{i, j}$, called a cell, denotes the matrix with exactly one nonzero entry, that being a one in the $(i, j)$ entry. Let $R_{i}$ denote the matrix whose $i^{\text {th }}$ row is all ones and is zero elsewhere, and $C_{j}$ denote the matrix whose $j^{\text {th }}$ column is all ones and is zero elsewhere. We let $|A|$ denote the number of nonzero entries in the matrix $A$.

Definition 1.4. Let $\mathcal{R}$ be the field of reals, let $\mathcal{F}=\{\alpha \in \mathcal{R} \mid 0 \leq \alpha \leq 1\}$ denote a subset of reals. Define $a+b=\max \{a, b\}$ and $a \cdot b=\min \{a, b\}$ for all a,b in $\mathcal{F}$. Then $(\mathcal{F},+, \cdot)$ is called a fuzzy semiring. Let $\mathcal{M}_{m, n}(\mathcal{F})$ denote the set of all $m \times n$ matrices with entries in a fuzzy semiring $\mathcal{F}$. We call a matrix in $\mathcal{M}_{m, n}(\mathcal{F})$ as a fuzzy matrix.

Definition 1.5. A line of a matrix $A$ is a row or a column of the matrix $A$.

Definition 1.6. A matrix $A \in \mathcal{M}_{m, n}(\mathcal{S})$ has term rank $k(t(A)=k)$ if the least number of lines needed to include all nonzero elements of $A$ is equal to $k$. Let us denote by $c(A)$ the least number of columns needed to include all nonzero elements of $A$ and by $r(A)$ the least number of rows needed to include all nonzero elements of $A$. 
Definition 1.7. A matrix $A \in \mathcal{M}_{m, n}(\mathcal{S})$ has zero-term rank $k(z(A)=k)$ if the least number of lines needed to include all zero elements of $A$ is equal to $k$.

Example 1.8. Let

$$
A=\left(\begin{array}{ccc}
1 & \frac{1}{2} & \frac{1}{3} \\
\frac{1}{2} & 0 & \frac{1}{4} \\
1 & \frac{1}{3} & \frac{1}{2}
\end{array}\right), \quad B=\left(\begin{array}{ccc}
1 & 0 & 0 \\
\frac{1}{2} & \frac{1}{2} & 0 \\
0 & 0 & 0
\end{array}\right)
$$

be matrices over fuzzy semiring $\mathcal{F}$. Then $z(A)=1$ and $z(B)=3$.

Definition 1.9. A matrix $A \in \mathcal{M}_{m, n}(\mathcal{S})$ has factor rank $k(\operatorname{rank}(A)=k)$ if there exist matrices $B \in \mathcal{M}_{m, k}(\mathcal{S})$ and $C \in \mathcal{M}_{k, n}(\mathcal{S})$ such that $A=B C$ and $k$ is the smallest positive integer such that such a factorization exists. By definition the only matrix with factor rank equal to 0 is the zero matrix, $O$.

If $\mathcal{S}$ is a subsemiring of a certain field then there is a usual rank function $\rho(A)$ for any matrix $A \in \mathcal{M}_{m, n}(\mathcal{S})$. It is easy to see that these functions are not equal in general but the inequality $\operatorname{rank}(A) \geq \rho(A)$ always holds.

Example 1.10. Consider $z_{+}$, the set of nonnegative integers. The semiring $z_{+}$is embedded in the real field $\mathcal{R}$. Then the matrix

$$
A=\left(\begin{array}{lll}
0 & 1 & 2 \\
2 & 1 & 0 \\
3 & 3 & 3
\end{array}\right)
$$

has different values as, where $\operatorname{rank}(A)=3$ and $\rho(A)=2$.

Definition 1.11. Let $\mathcal{F}$ be a fuzzy semiring. An operator $T: \mathcal{M}_{m, n}(\mathcal{F}) \rightarrow \mathcal{M}_{m, n}(\mathcal{F})$ is called linear if $T(X+Y)=T(X)+T(Y)$ and $T(\alpha X)=\alpha T(X)$ for all $X, Y \in \mathcal{M}_{m, n}(\mathcal{F}), \alpha \in \mathcal{F}$.

Definition 1.12. We say an operator, $T$, preserves a set $\mathcal{P}$ if $X \in \mathcal{P}$ implies that $T(X) \in \mathcal{P}$, or, if $(X, Y) \in \mathcal{P}$ implies that $(T(X), T(Y)) \in \mathcal{P}$ when $\mathcal{P}$ is a set of ordered pairs.

Definition 1.13. An operator $T$ strongly preserves the set $\mathcal{P}$ if $X \in \mathcal{P}$ if and only if $T(X) \in \mathcal{P}$, or, if $(X, Y) \in \mathcal{P}$ if and only if $(T(X), T(Y)) \in \mathcal{P}$ when $\mathcal{P}$ is a set of ordered pairs.

Definition 1.14. The matrix $X \circ Y$ denotes the Hadamard or Schur product, i.e., the $(i, j)$ entry of $X \circ Y$ is $x_{i, j} y_{i, j}$.

Definition 1.15. An operator $T$ is called a $(P, Q, B)$-operator if there exist permutation matrices $P$ and $Q$, and a matrix $B$ with no zero entries, such that $T(X)=P(X \circ B) Q$ for all $X \in \mathcal{M}_{m, n}(\mathcal{F})$, or, if $m=n, T(X)=P(X \circ B)^{t} Q$ for all $X \in \mathcal{M}_{m, n}(\mathcal{F})$. A $(P, Q, B)$-operator is called a $(P, Q)$-operator if $B=J$, the matrix of all ones. 
It was shown in [2] and [4] that linear preservers for extremal cases of classical matrix inequalities over fields are types of $(P, Q)$-operators where $P$ and $Q$ are arbitrary invertible matrices. On the other side, linear preservers for various rank functions over semirings have been the object of much study during the last years(see for example [6]), in particular term rank and zero term rank were investigated in the last few years(see for example [5]).

Definition 1.16. We say that the matrix $A$ dominates the matrix $B$ if and only if $b_{i, j} \neq 0$ implies that $a_{i, j} \neq 0$, and we write $A \geq B$ or $B \leq A$.

Definition 1.17. If $A$ and $B$ are matrices and $A \geq B$ we let $A \backslash B$ denote the matrix $C$ where

$$
c_{i, j}=\left\{\begin{array}{rr}
0 & \text { if } b_{i, j} \neq 0 \\
a_{i, j} & \text { otherwise }
\end{array}\right.
$$

The behavior of the function $\rho$ with respect to matrix multiplication and addition is given by the following inequalities:

The rank-sum inequalities:

$$
|\rho(A)-\rho(B)| \leq \rho(A+B) \leq \rho(A)+\rho(B) ;
$$

Sylvester's laws:

$$
\rho(A)+\rho(B)-n \leq \rho(A B) \leq \min \{\rho(A), \rho(B)\} ;
$$

and the Frobenius inequality:

$$
\rho(A B)+\rho(B C) \leq \rho(A B C)+\rho(B)
$$

where $A, B$ and $C$ are conformal matrices with coefficients from a field.

\section{Zero-term rank inequality over fuzzy semiring}

We obtain inequalities for the zero-term rank addition over fuzzy semirings. We also show that these inequalities are exact and best possible.

Proposition 2.1. Let $\mathcal{F}$ be a fuzzy semiring. For $A, B \in \mathcal{M}_{m, n}(\mathcal{F})$ one has that $0 \leq z(A+B) \leq \min \{z(A), z(B)\}$. These bounds are exact and the best possible.

Proof. The lower bound follows from the definition of the zero-term rank function.

In order to check that this exact and the best possible for each pair $(r, s), 0 \leq$ $r, s \leq \min \{m, n\}$ let us consider the family of matrices $A_{r}=J \backslash\left(\sum_{i=1}^{r} E_{i, i}\right), B_{s}=$ $J \backslash\left(\sum_{i=1}^{s} E_{i, i+1}\right)$ if $s<\min \{m, n\}$ and $B_{s}=J \backslash\left(\Sigma_{i=1}^{s-1} E_{i, i+1}+E_{s, l}\right)$ if $s=\min \{m . n\}$. Then $z\left(A_{r}\right)=r, z\left(B_{s}\right)=s$ by definition and $z\left(A_{r}+B_{s}\right)=0$ by antinegativity.

The upper bound follows directly from the definition of zero-term rank and from the antinegativity of $\mathcal{F}$. For the proof of its exactness let us take $A=J$ and $B=O$. In order to check that this bound is the best possible we consider the following family of matrices: for each pair $(r, s), 0 \leq r, s \leq \min \{m, n\}$ let us consider the 
matrices $A_{r}=J \backslash\left(\sum_{i=1}^{r} E_{i, i}\right)$ and $B_{s}=J \backslash\left(\sum_{i=1}^{s} E_{i, i}\right)$.

Proposition 2.2. Let $\mathcal{F}$ be a fuzzy semiring. For $A \in \mathcal{M}_{m, n}(\mathcal{F}), B \in \mathcal{M}_{n, k}$ one has that $0 \leq z(A B) \leq \min \{z(A)+z(B), k, m\}$. These bounds are exact and the best possible for $n>2$.

Proof. The lower bound follows from the definition of the zero-term rank function. In order to show that this bound is exact and the best possible let us consider the family of matrices: for each pair $(r, s), 0 \leq r \leq \min \{m, n\}, 0 \leq s \leq \min \{k, n\}$, we take $A_{r}=J \backslash\left(\sum_{i=1}^{s} E_{i, i}\right), B_{s}=J \backslash\left(\sum_{i=1}^{s} E_{i, i+1}\right)$ if $s<\min \{k, n\}$ and $B_{s}=$ $J \backslash\left(\Sigma_{i=1}^{s-1} E_{i, i+1}+E_{s, 1}\right)$ if $s=\min \{k, n\}$. Then $z\left(A_{r}\right)=r, z\left(B_{s}\right)=s$ by definition and if $n>2$ then $A_{r} B_{s}$ does not have zero elements by antinegativity. Thus $z\left(A_{r} B_{s}\right)=0$.

The upper bound follows directly from the definition of zero-term rank and from the antinegativity of $\mathcal{F}$.

In order to show that this bound is exact and the best possible let us consider the family of matrices: for each pair $(r, s), 0 \leq r \leq \min \{m, n\}, 0 \leq s \leq \min \{k, n\}$, we take $A_{r}=J \backslash\left(\sum_{i=1}^{r} R_{i}\right)$ and $B_{s}=J \backslash\left(\sum_{i=1}^{s} C_{i}\right)$.

Example 2.3. The triple $\left(C_{1}, I, R_{1}\right)$ is a counterexample to the zero-term rank version of the Frobenius inequality, since

$$
z\left(C_{1}\right)+z\left(R_{1}\right)=2 n-2>z\left(C_{1} R_{1}\right)+z(I)=n
$$

for $n>2$.

\section{Basic results for linear operators on fuzzy semiring}

In this section, we obtain some basic results for our main theorems in the section 4. For a surjective linear operator, we have the followings.

Theorem 3.1. Let $\mathcal{F}$ be a fuzzy semiring and $T: \mathcal{M}_{m, n}(\mathcal{F}) \rightarrow \mathcal{M}_{m, n}(\mathcal{F})$ be a linear operator. Then the following are equivalent:

1. $T$ is bijective,

2. $T$ is surjective,

3. There exists a permutation $\sigma$ on $\{(i, j) \mid i=1,2, \cdots, m ; j=1,2, \cdots, n\}$ such that $T\left(E_{i, j}\right)=E_{\sigma(i, j)}$.

Proof. That 1) implies 2) and 3) implies 1) is straight forward. We now show that 2) implies 3).

We assume that $T$ is surjective. Then, for any pair $(i, j)$, there exists some $X$ such that $T(X)=E_{i, j}$. Clearly $X \neq O$ by the linearity of $T$. Thus there is a pair of indexes $(r, s)$ such that $X=x_{r, s} E_{r, s}+X^{\prime}$ where $(r, s)$ entry of $X^{\prime}$ is zero and the following two conditions are satisfied: $x_{r, s} \neq 0$ and $T\left(E_{r, s}\right) \neq O$. Indeed, if in 
the contrary for all pairs $(r, s)$ either $x_{r, s}=0$ or $T\left(E_{r, s}\right)=O$ then $T(X)=0$ which contradicts with the assumption $T(X)=E_{i, j} \neq 0$. Hence

$$
T\left(x_{r, s} E_{r, s}\right) \leq T\left(x_{r, s} E_{r, s}\right)+T\left(X \backslash\left(x_{r, s} E_{r, s}\right)\right)=T(X)=E_{i, j} .
$$

That is, $x_{r, s} T\left(E_{r, s}\right)=T\left(x_{r, s} E_{r, s}\right) \leq E_{i, j}$. Thus $T\left(x_{r, s} E_{r, s}\right)=\alpha E_{i, j}$ for a certain $\alpha \in \mathcal{F}$. That is there is some permutaion $\sigma$ on $\{(i, j) \mid i=1,2, \cdots, m ; j=$ $1,2, \cdots, n\}$ such that for some scalars $b_{i, j}, T\left(E_{i, j}\right)=b_{i, j} E_{\sigma(i, j)}$. we now only need show that the $b_{i, j}$ are all units. Since $T$ is surjective and $T\left(E_{r, s}\right) \not \leq E_{\sigma(i, j)}$ for $(r, s) \neq(i, j)$, there is some $\alpha$ such that $T\left(\alpha E_{i, j}\right)=E_{\sigma(i, j)}$. But then, since $T$ is linear, $T\left(\alpha E_{i, j}\right)=\alpha T\left(E_{i, j}\right)=\alpha b_{i, j} E_{\sigma(i, j)}=E_{\sigma(i, j)}$. That is, $\alpha b_{i, j}=1$, or $b_{i, j}$ is a unit. But 1 is the only unit over fuzzy semiring.

Lemma 3.2 Let $\mathcal{F}$ be a fuzzy semiring, $T: \mathcal{M}_{m, n}(\mathcal{F}) \rightarrow \mathcal{M}_{m, n}(\mathcal{F})$ be an operator which maps lines to lines and is defined by $T\left(E_{i, j}\right)=E_{\sigma(i, j)}$, where $\sigma$ is a permutation on the set $\{(i, j) \mid i=1,2, \cdots, m ; j=1,2, \cdots, n\}$. Then $T$ is a $(P, Q)$-operator.

Proof. Since no combination of $u$ rows and $v$ columns can dominate $J$ where $u+v=m$ unless $v=0$ (or if $m=n$, if $u=0$ ) we have that either the image of each row is a row and the image of each column is a column, or $m=n$ and the image of each row is a column and the image of each column is a row. Thus, there are permutation matrices $P$ and $Q$ such that $T\left(R_{i}\right) \leq P R_{i} Q$ and $T\left(C_{j}\right) \leq P C_{j} Q$ or, if $m=n, T\left(R_{i}\right) \leq P\left(R_{i}\right)^{t} Q$ and $T\left(C_{j}\right) \leq P\left(C_{j}\right)^{t} Q$. Since each cell lies in the intersection of a row and a column and $T$ maps nonzero cells to nonzero (weighted) cells, it follows that $T\left(E_{i, j}\right)=P E_{i, j} Q$, or, if $m=n, T\left(E_{i, j}\right)=P E_{j, i} Q=P\left(E_{i, j}\right)^{t} Q$.

\section{The zero-term rank preservers over fuzzy semiring}

In this section, we obtain the characterizations of the linear operators that preserve the set of matrix pairs which arise as the extremal cases in the inequalities of zero-term rank of matrix sums.

Below, we use the following notations in order to denote sets of matrices that arise as extremal cases in the inequalities of zero-term rank of matrix sums listed in section 3.

$$
\begin{gathered}
z_{1}(\mathcal{F})=\left\{(X, Y) \in \mathcal{M}_{m, n}(\mathcal{F})^{2} \mid z(X+Y)=\min \{z(X), z(Y)\}\right\} ; \\
z_{2}(\mathcal{F})=\left\{(X, Y) \in \mathcal{M}_{m, n}(\mathcal{F})^{2} \mid z(X+Y)=0\right\} .
\end{gathered}
$$

\subsection{Linear preservers of $z_{1}(\mathcal{F})$}

Consider the set of matrix pairs:

$$
Z_{1}(\mathcal{F})=\left\{(X, Y) \in \mathcal{M}_{m, n}(\mathcal{F})^{2} \mid z(X+Y)=\min \{z(X), z(Y)\}\right\} .
$$

We characterize the linear operators that preserve the set $z_{1}(\mathcal{F})$. 
Example 4.1. Let

$$
X=\left(\begin{array}{ccc}
\frac{1}{2} & 0 & 1 \\
\frac{1}{3} & 0 & \frac{1}{2} \\
1 & 0 & \frac{1}{2}
\end{array}\right), \quad Y=\left(\begin{array}{ccc}
1 & 0 & \frac{1}{2} \\
\frac{1}{2} & 0 & \frac{1}{3} \\
\frac{1}{3} & 0 & \frac{1}{2}
\end{array}\right)
$$

be matrices over fuzzy semiring $\mathcal{F}$. Then $z(X+Y)=z\left(\left(\begin{array}{ccc}1 & 0 & 1 \\ \frac{1}{2} & 0 & \frac{1}{2} \\ 1 & 0 & \frac{1}{2}\end{array}\right)\right)=1$ while $z(X)=z(Y)=1$. Therefore $(X, Y) \in Z_{1}(\mathcal{F})$ and hence $z_{1}(\mathcal{F})$ is not empty.

Theorem 4.2. Let $\mathcal{F}$ be a fuzzy semiring, $T: \mathcal{M}_{m, n}(\mathcal{F}) \rightarrow \mathcal{M}_{m, n}(\mathcal{F})$ be a surjective linear map. Then $T$ preserves the set $z_{1}(\mathcal{F})$ if and only if $T$ is a $(P, Q)$-operator, where $P$ and $Q$ is a permutation matrices of appropriate sizes.

Proof. By Theorem 3.1 we have that $T\left(E_{i, j}\right)=E_{\sigma(i, j)}$ for all $i, j, 1 \leq i \leq m$, $1 \leq j \leq n$, where $\sigma$ is a permutation on the set of pairs $(i, j)$.

Let us show that $T$ maps lines to lines. Suppose that the images of two cells are not in the same line, but the cells are, say $E_{i, j}, E_{i, k}$ are the cells such that $T\left(E_{i, j}\right), T\left(E_{i, k}\right)$ are not in the same line. Then one has that $z\left(\left(J \backslash E_{i, j} \backslash E_{i, k}\right)+\right.$ $\left.E_{i, k}\right)=1=z\left(J \backslash E_{i, j} \backslash E_{i, k}\right)$, i.e. $\left(J \backslash E_{i, j} \backslash E_{i, k}, E_{i, k}\right) \in Z_{1}$, as far as

$$
z\left(T\left(J \backslash E_{i, j} \backslash E_{i, k}\right)+T\left(E_{i, k}\right)\right)=1<2=\min \left\{z\left(T\left(J \backslash E_{i, j} \backslash E_{i, k}\right)\right), z\left(T\left(E_{i, k}\right)\right\},\right.
$$

i.e. $\left(T\left(J \backslash E_{i, j} \backslash E_{i, k}\right), T\left(E_{i, k}\right)\right) \notin z_{1}$, a contradiction. Thus $T$ maps lines to lines. By Lemma 3.2 it follows that $T$ is a $(P, Q)$-operator where $P$ and $Q$ are permutation matrices of appropriate sizes.

Conversely, if $(X, Y) \in z_{1}$ then $z(X+Y)=z(X)$, and hence $z(P(X+Y) Q)=$ $z(P X Q)$, and $z(T(X+Y))=z(T(X))$. Hence $(T(X), T(Y)) \in z_{1}$. That is, $T$ preserves $z_{1}$.

\subsection{Linear preservers of $z_{2}(\mathcal{F})$}

Consider the set of matrix pairs:

$$
Z_{2}(\mathcal{F})=\left\{(X, Y) \in \mathcal{M}_{m, n}(\mathcal{F})^{2} \mid z(X+Y)=0\right\} .
$$

We characterize the linear operators that preserve the set $z_{2}(\mathcal{F})$.

Example 4.3. Let

$$
X=\left(\begin{array}{ccc}
\frac{1}{2} & 0 & 1 \\
\frac{1}{3} & 0 & \frac{1}{2} \\
1 & 0 & \frac{1}{2}
\end{array}\right), \quad Y=\left(\begin{array}{ccc}
1 & \frac{1}{2} & 0 \\
\frac{1}{2} & \frac{1}{2} & 0 \\
\frac{1}{3} & \frac{1}{2} & 0
\end{array}\right)
$$

be matrices over fuzzy semiring $\mathcal{F}$. Then $z(X+Y)=z\left(\left(\begin{array}{ccc}1 & \frac{1}{2} & 1 \\ \frac{1}{2} & \frac{1}{2} & \frac{1}{2} \\ 1 & \frac{1}{2} & \frac{1}{2}\end{array}\right)\right)=0$.

Therefore $(X, Y) \in z_{2}(\mathcal{F})$ and hence $z_{2}(\mathcal{F})$ is not empty. 
Theorem 4.4. Let $\mathcal{F}$ be a fuzzy semiring, $T: \mathcal{M}_{m, n}(\mathcal{F}) \rightarrow \mathcal{M}_{m, n}(\mathcal{F})$ be a surjective linear map. Then $T$ preserves the set $Z_{2}(\mathcal{F})$ if and only if $T$ is a $(P, Q)$-operator, where $P$ and $Q$ is a permutation matrices of appropriate sizes.

Proof. By Theorem 3.1 we have that $T\left(E_{i, j}\right)=E_{\sigma(i, j)}$ for all $i, j, 1 \leq i \leq m$, $1 \leq j \leq n$, where $\sigma$ is a permutation on the set of pairs $\{(i, j) \mid 1 \leq i \leq m, 1 \leq j \leq n\}$.

Let us show that $T$ maps lines to lines. For all $i=1,2, \ldots, n$, let $C_{1}+\ldots+$ $C_{n-1}=X, Y=C_{n}$. Then $z(X+Y)=z(J)=0$. Hence $z(T(X)+T(Y))=0$ by assumption. Thus each column is mapped under $T$ to a column. Similarly, each row is mapped under $T$ to a row. Thus $T$ maps lines to lines.

By Lemma 3.2 it follows that $T$ is a $(P, Q)$-operator where $P$ and $Q$ are permutation matrices of appropriate sizes.

Conversely, if $z(X+Y)=0$, then sets of zero cells in $X$ and $Y$ are disjoint. Thus the same holds for $T(X)$ and $T(Y)$ since $\sigma$ is a permutation on the set of pairs $\{(i, j) \mid 1 \leq i \leq m, 1 \leq j \leq n\}$. Hence in $(T(X)+T(Y))$ there is no zero elements. i.e. $z(T(X)+T(Y))=0$. Thus $(P, Q)$-operator preserves the set $z_{2}(\mathcal{F})$.

As a concluding remark, we have characterized the linear operators that preserve the extreme sets of the zero-term rank inequalities of the matrix sums over fuzzy semirings. For further research, we hope to study the zero-term rank inequalities of matrix product over fuzzy semirings.

\section{References}

[1] L. B. Beasley and A. E. Guterman, Rank inequalities over semirings, J. Korean Math. Soc., 42(2)(2005), 223-241.

[2] L. B. Beasley, A. E. Guterman, and C. L. Neal, Linear preservers for Sylvester and Frobenius bounds on matrix rank, Rocky Mountains J. Math., 36(1)(2006), 67-75.

[3] L. B. Beasley, A. E. Guterman, Y. B. Jun and S. Z. Song, Linear preservers of extremes of rank inequalities over semirings: Row and Column ranks, Linear Algebra Appl., 413(2006), 495-509.

[4] L. B. Beasley, S.-G. Lee, and S.-Z. Song, Linear operators that preserve pairs of matrices which satisfy extreme rank properties, Linear Algebra Appl., 350(2002), 263-272.

[5] L. B. Beasley, S.-G. Lee, S.-Z. Song, Linear operators that preserve zero-term rank of Boolean matrices, J. Korean Math. Soc., 36(6)(1999), 1181-1190.

[6] P. Pierce and others, A Survey of Linear Preserver Problems, Linear and Multilinear Algebra, 33(1992), 1-119.

[7] S. Z. Song, Topics on linear preserver problems - a brief introduction, (Korean), Commun. Korean Math. Soc., 21(2006), 595-612. 